\title{
Physical evaluation of an ultra-high-resolution CT scanner
}

\author{
Luuk J. Oostveen $^{1}$ (D) $\cdot$ Kirsten L. Boedeker ${ }^{2} \cdot$ Monique Brink $^{1} \cdot$ Mathias Prokop ${ }^{1} \cdot$ Frank de Lange ${ }^{1}$. \\ Ioannis Sechopoulos ${ }^{1}$
}

Received: 28 June 2019 /Revised: 16 November 2019 / Accepted: 16 December 2019/Published online: 10 February 2020

(C) The Author(s) 2020

\begin{abstract}
Objectives To evaluate the technical performance of an ultra-high-resolution CT (UHRCT) system.

Methods The physico-technical capabilities of a novel commercial UHRCT system were assessed and compared with those of a current-generation multi-detector (MDCT) system. The super-high-resolution (SHR) mode of the system uses $0.25 \mathrm{~mm}$ (at isocentre) detector elements (dels) in the in-plane and longitudinal directions, while the high-resolution (HR) mode bins two dels in the longitudinal direction. The normal-resolution (NR) mode bins dels $2 \times 2$, resulting in a del-size equivalent to that of the MDCT system. In general, standard procedures and phantoms were used to perform these assessments.

Results The UHRCT MTF (10\% MTF $4.1 \mathrm{lp} / \mathrm{mm}$ ) is twice as high as that of the MDCT (10\% MTF $1.9 \mathrm{lp} / \mathrm{mm})$, which is comparable to the MTF in the NR mode (10\% MTF $1.7 \mathrm{lp} / \mathrm{mm}$ ). The width of the slice sensitivity profile in the SHR mode (FWHM $0.45 \mathrm{~mm}$ ) is about $60 \%$ of that of the MDCT (FWHM $0.77 \mathrm{~mm}$ ). Uniformity and CT numbers are within the expected range. Noise in the high-resolution modes has a higher magnitude and higher frequency components compared with MDCT. Low-contrast visibility is lower for the NR, HR and SHR modes compared with MDCT, but about a 14\%, for NR, and 23\%, for HR and SHR, dose increase gives the same results. Conclusions HR and SHR mode scanning results in double the spatial resolution, with about a $23 \%$ increase in dose required to achieve the same low-contrast detectability.

Key Points

- Resolution on UHRCT is up to twice as high as for the tested MDCT.

-With abdominal settings, UHRCT needs higher dose for the same low-contrast detectability as MDCT, but dose is still below achievable levels as defined by current diagnostic reference levels.

- The UHRCT system used in normal-resolution mode yields comparable resolution and noise characteristics as the MDCT system.
\end{abstract}

Keywords Physics $\cdot$ Phantoms $\cdot$ Imaging $\cdot$ Multi-detector computed tomography

\author{
Abbreviations \\ ACR American College of Radiology \\ AI Artificial intelligence \\ $\mathrm{CTDI}_{\mathrm{vol}} \quad$ Volume CT dose index \\ Del Detector element
}

Electronic supplementary material The online version of this article (https://doi.org/10.1007/s00330-019-06635-5) contains supplementary material, which is available to authorized users.

Luuk J. Oostveen

Luuk.Oostveen@radboudumc.nl

1 Department of Radiology and Nuclear Medicine, Radboud University Medical Center, P.O. Box 9101 (Route 766), 6500 HB Nijmegen, The Netherlands

2 Canon Medical Systems Corporation, Otawara, Japan

$\begin{array}{ll}\text { ESF } & \text { Edge spread function } \\ \text { FWHM } & \text { Full width at half maximum } \\ \text { HR } & \text { High resolution } \\ \text { LSF } & \text { Line spread function } \\ \text { MTF } & \text { Modulation transfer function } \\ \text { NPS } & \text { Noise power spectrum } \\ \text { NR } & \text { Normal resolution } \\ \text { SHR } & \text { Super-high resolution } \\ \text { SSP } & \text { Slice sensitivity profile } \\ \text { UHRCT } & \text { Ultra-high-resolution CT }\end{array}$

\section{Introduction}

Advances in multi-detector computed tomography (MDCT) technology have continued over recent years. New detector hardware has resulted in the introduction of wider detectors 
[1], new electronic designs with lower noise [2] and, more recently, smaller detector elements [3]. The ability to visualise anatomy and pathology in greater detail has the potential to benefit the imaging of the lung, temporal bone, vasculature and stent structure, as well as visualisation of small tumours and structures [3-6]. Ultra-high spatial resolution capabilities may also lead to a reduction in artefacts such as blooming, as well as an increased ability to quantify features of anatomical and pathological structures.

While many factors, e.g. focal spot size and number of views, impact the final spatial resolution of a CT image, the maximum spatial resolution of a CT system is fundamentally dictated by the Nyquist frequency of the detector in both the midplane and longitudinal dimensions. Several approaches to reducing the detector element size in $\mathrm{CT}$ have been explored in the past. First, flat panel prototype volume CT scanners were constructed, but they suffered from poor low-contrast detectability and gantry rotation speed limitations preventing commercial implementation [7-10]. A second approach was the introduction of a tantalum grid over a selected number of detector elements in a conventional CT system, in order to reduce the active detector element area, yielding $250 \mu \mathrm{m}$ resolution images. However, this approach inherently decreased the dose efficiency, limiting its utility as a general clinical system [11]. Third, a prototype fine-cell CT scanner was developed using a $312.5-\mu \mathrm{m}$ channel thickness that achieved $27.6 \mathrm{lp} / \mathrm{cm}$ at $2 \%$ of the MTF, but with high noise [12]. Finally, photon-counting detectors permitted the measurement of individual photons and their corresponding energy, ideally eliminating the need for detector septa and grids. An ultrahigh-resolution mode on a prototype photon-counting system generated by grouping detector elements into a $2 \times 2$ formation rather than a $4 \times 4$ formation for conventional resolution resulted in $250 \mu \mathrm{m} \times 250 \mu \mathrm{m}$ resolution at isocentre [13]. Currently, however, the commercial development of photoncounting scanners is hampered by pulse pileup and other technical issues [14].

Recently, an ultra-high-resolution CT system (UHRCT; Aquilion Precision, Canon Medical Systems Corporation) was brought to market with a detector element size of $0.25 \mathrm{~mm}$ at isocentre. The purpose of this work is to perform a fundamental physics assessment of this UHRCT system, and compare it with a conventional CT system.

\section{Materials and methods}

For this evaluation, we compared the characteristics of the UHRCT system to a current MDCT system, with comparable hardware, geometry and reconstruction modes, but without the high spatial resolution capability. The following characteristics were measured: spatial resolution, CT number accuracy, uniformity, low-contrast detectability and noise. All measurements were made using abdominal imaging protocols.

\section{CT scanner and acquisition}

The UHRCT system used has been previously described [3]. Briefly, this system has three resolution modes: normal resolution (NR), high resolution (HR) and super-high resolution (SHR). In NR mode, the $0.25 \mathrm{~mm}$ detector elements, at isocentre, are read out in $2 \times 2$ binned mode. The detector element size is therefore $0.5 \mathrm{~mm}$ in-plane as well as in the longitudinal direction, comparable to current MDCT systems. In HR mode, the in-plane element size halves while in the longitudinal direction it remains the same as in NR mode. Finally, in SHR mode, the native detector element size of $0.25 \mathrm{~mm}$ in both directions is used. Therefore, the in-plane resolutions of the HR and the SHR modes are the same, while the resolution in the longitudinal direction for the latter is twice as high as that of the former. The UHRCT system has various focal spot sizes, the smallest being of nominal size $0.4 \times 0.5 \mathrm{~mm}^{2}$. This focal spot size can be used in all resolution modes up to a tube current of $260 \mathrm{~mA}$. The matrix size can have dimensions of $512 \times 512,1024 \times 1024$ or $2048 \times$ 2048 pixels, with the latter two sizes not being available in NR mode.

For reference, measurements were performed on a current MDCT system (Aquilion One Genesis, Canon Medical Systems Corporation) with a fixed detector element size of $0.5 \mathrm{~mm}$ in both directions. The smallest nominal focal spot size of this system is $0.9 \times 0.8 \mathrm{~mm}^{2}$.

The default acquisition parameters for this evaluation are given in Table 1. These parameters mimic an abdominal CT protocol as used at our institution. As stated in Table 1, all acquisitions were reconstructed using hybrid-iterative reconstruction (AIDR 3D Enhanced, FC08). The only exception is the measurement of the maximum spatial resolution, which was performed using filtered back projection and a highresolution kernel (FC90). In all cases, the same reconstruction technique was used on both $\mathrm{CT}$ systems.

\section{Spatial resolution}

The maximum spatial resolution that the UHRCT can produce was determined by measuring the modulation transfer function (MTF) using a sequential acquisition mode and filtered backprojection reconstruction. A $50-\mu \mathrm{m}$ diameter tungsten wire fixed in a frame of balsa wood was imaged. The wire was positioned approximately $1.5 \mathrm{~cm}$ above the isocentre and scanned using the sequential scan mode. In this mode, only the central 4 detector slices are used and combined in one reconstructed slice. A 20-mm FOV was reconstructed using filtered backprojection and a high-resolution reconstruction 
Table 1 Default parameters for the measurements performed. Deviation from these parameters for each test are noted in the corresponding test descriptions

\begin{tabular}{lll}
\hline Parameter & UHRCT & MDCT \\
\hline Tube voltage $(\mathrm{kVp})$ & 120 & 120 \\
Tube current $(\mathrm{mA})$ & 260 & 270 \\
Effective tube current time product (mAs) & 160 & 166 \\
Computed tomography dose index volume (mGy) & NR 9.1 & 9.2 \\
& HR 11.2 & \\
Rotation time (s) & SHR 11.3 & 0.5 \\
Focal spot size (mm $\left.{ }^{2}\right)$ & 0.5 & $0.9 \times 0.8$ \\
Scan mode, collimation $(\mathrm{mm})$ & $0.4 \times 0.5$ & Helical, \\
& Helical, & $80 \times 0.50$ \\
Pitch & NR/HR $80 \times 0.50$ & \\
Field of view (mm) & SHR $160 \times 0.25$ & 0.813 \\
Reconstruction method & 0.813 & 500 \\
Reconstruction kernel & 500 & AIDR 3D enhanced \\
Reconstruction matrix & AIDR 3D enhanced & FC08 \\
Slice thickness, increment $(\mathrm{mm})$ & FC08 & $512 \times 512$ \\
& NR 512 $\times 512$ & \\
& HR/SHR $1024 \times 1024$ & $0.5,0.5$ \\
& SHR $0.25,0.25$ & \\
\hline
\end{tabular}

kernel (FC90). The tube current was set at $100 \mathrm{~mA}$. The MTF was calculated as described in Appendix A.

For the resolution measurements using hybrid-iterative reconstruction, the tungsten wire cannot be used as this reconstruction technique (AIDR 3D Enhanced) diminishes the delta pulse of such a thin wire. Therefore, the Teflon rod in the CTP401 module of the Catphan 500 phantom [15] was used. The phantom was placed such that the Teflon rod was laterally centred at five different vertical positions: at the isocentre and at $5 \mathrm{~cm}, 10 \mathrm{~cm}, 15 \mathrm{~cm}$ and $20 \mathrm{~cm}$ above the isocentre. Again, further details on the MTF calculation are provided in Appendix A.

Even though the same hybrid-iterative reconstruction algorithm and kernel are available on both systems, to exclude any possible differences that they might have internally, the MTF in the isocentre was also determined using filtered backprojection with the same kernel (FC08).

\section{Slice sensitivity profile}

The spatial resolution in the longitudinal direction was measured using the slice sensitivity profile (SSP). For this, a $0.025-\mathrm{mm}$ thick tungsten foil embedded in a polyurethane cylinder was scanned in the isocentre using the default settings as given in Table 1 and hybrid-iterative reconstruction. A 40$\mathrm{mm}$ FOV was reconstructed using a slice thickness of $0.25 \mathrm{~mm}$ and a slice increment of $0.1 \mathrm{~mm}$. Using ImageJ (version 1.52d, National Institutes of Health), the average value within a circular ROI with a 23 voxel diameter located inside the disc was plotted against the longitudinal distance.
From the SSP, the full width at half maximum (FWHM) was calculated.

\section{CT number uniformity}

Uniformity was measured using a 320-mm water phantom similar to the method proposed by the American College of Radiology (ACR) [16]. Five circular ROIs with a diameter of $32 \mathrm{~mm}$ were selected in the central slice, at the centre and at the 3, 6, 9 and 12 o'clock positions. The mean pixel HU values of the peripheral ROIs were compared with the central one.

\section{Noise}

Noise appearance was measured by calculating the noise power spectrum (NPS) for different conditions using a cylindrical water phantom with a diameter of $320 \mathrm{~mm}$. Default acquisition settings were used. Per setting, 80 scans with an $8-\mathrm{cm}$ scan range were acquired. Three slices per scan, separated by at least 15 slices to minimise noise correlation, were used for the NPS calculation to improve statistics. The NPS was calculated using the method described in Appendix A. As a measure of the noise magnitude, the standard deviation was calculated in the same area.

\section{CT number accuracy}

For the determination of the CT numbers, the Catphan CTP401 module [15] was imaged using the default parameters, reconstructed with $10 \mathrm{~mm}$ thick slices. For the CT 
number of water, one of the NPS acquisitions was used. CT numbers were measured as the mean value over a circular ROI with a diameter of $1 \mathrm{~cm}$ using ImageJ and compared with the range given in the ACR CT quality control manual [16].

\section{Low-contrast visibility}

Low contrast can be affected by higher resolution and inherently higher noise at the same dose level. Therefore, the lowcontrast objects in the Catphan CTP515 module were imaged using the default parameters in all three modes. As the CTDI ${ }_{\mathrm{vol}}$ is higher in the SHR and HR modes for the same tube settings, the tube current was lowered to $210 \mathrm{~mA}$ to get the same $\mathrm{CTDI}_{\mathrm{vol}}$ as for the NR mode. Reconstructions were made with $10 \mathrm{~mm}$ slice thickness and $1 \mathrm{~mm}$ slice increment. One reader (LO) evaluated the middle slice of the CTP515 phantom using diagnostic monitors in a radiological reading room and determined the number of visible contrast objects for each contrast level. To determine the dose necessary to have the same lowcontrast visibility as with the MDCT, a series of acquisitions were made at progressively higher tube currents $(260 \mathrm{~mA}$, $280 \mathrm{~mA}, 300 \mathrm{~mA}, 330 \mathrm{~mA}, 370 \mathrm{~mA}$ and $400 \mathrm{~mA}$ ).

\section{Results}

\section{Spatial resolution}

Figure 1 and Table 2 show the MTFs of the UHRCT for the three modes and MDCT with filtered backprojection, representing the highest possible resolution. The spatial frequencies obtained with the UHRCT in SHR and HR modes are twice as high as those with the MDCT. The highest resolution of the UHRCT in NR mode is marginally lower than that of the MDCT. Using FBP in helical scan mode, the MTFs of the MDCT and the UHRCT in NR mode are equivalent.

The resolution change from the isocentre can be seen in Fig. 2. The edges of the Teflon rod are sharper in the isocentre. The MTFs resulting from these edges are plotted in Fig. 1. As
Table 2 Frequencies at which the MTF reaches 90\%, 50\%, 10\% and $2 \%$ for the UHRCT in SHR, HR and NR modes and for the MDCT

\begin{tabular}{lllll}
\hline MTF (\%) & \multicolumn{2}{l}{ Frequency $(\mathrm{lp} / \mathrm{mm})$} & \\
\cline { 2 - 5 } & \multicolumn{2}{l}{ UHRCT } & & MDCT \\
\cline { 2 - 5 } & SHR & HR & NR & \\
\hline 90 & 1.0 & 1.1 & 0.4 & 0.5 \\
50 & 2.6 & 2.7 & 1.0 & 1.2 \\
10 & 4.1 & 3.9 & 1.7 & 1.9 \\
2 & 4.8 & 5.2 & 2.9 & 2.2 \\
\hline
\end{tabular}

can be seen, the UHRCT MTF at the centre is about twice as high as that of the MDCT. Although the UHRCT MTF decreases continuously with increasing distance from the isocentre towards the periphery of the field of view, it remains higher than that of the MDCT, except at the farthest point measured: $20 \mathrm{~cm}$ from the isocentre in the radial direction.

\section{Slice sensitivity profile}

In HR mode, the SSP is comparable to the MDCT, having a FWHM of $0.79 \mathrm{~mm}$ and $0.77 \mathrm{~mm}$, respectively. For the UHRCT SHR mode, the SSP is narrower. The FWHM of the SHR mode, $0.45 \mathrm{~mm}$, is about 1.7 times smaller than that of the MDCT.

\section{CT number uniformity}

The differences of the CT number value of each peripheral ROI were within the $5 \mathrm{HU}$ limit from the centre ROI, as specified in the ACR protocol [16].

\section{Noise}

The noise magnitudes for the SHR, HR, NR and MDCT were (average \pm one standard deviation) $30.0 \mathrm{HU} \pm 0.3 \mathrm{HU}, 27.0$ $\mathrm{HU} \pm 0.3 \mathrm{HU}, 21.9 \mathrm{HU} \pm 0.3 \mathrm{HU}$ and $24.3 \mathrm{HU} \pm 0.3 \mathrm{HU}$, respectively. The NPS for the different modes are shown in
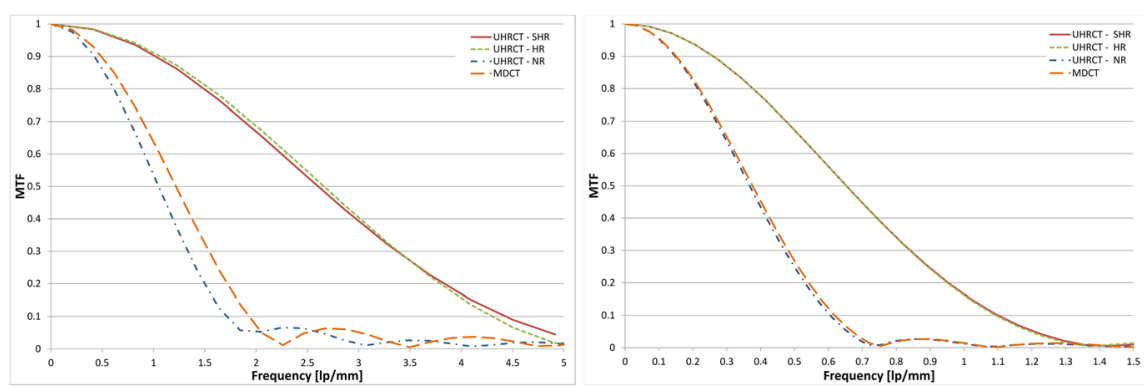

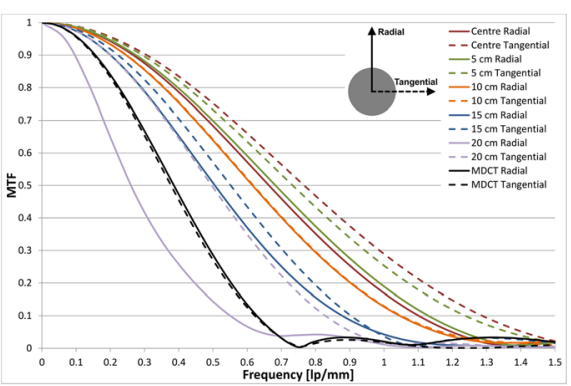

FBP (FC08). (Right) MTF of UHRCT under clinical conditions (AIDR3D, FC08) for different distances to the isocentre
Fig. 1 MTFs for UHRCT in SHR, HR and NR modes and MDCT. (Left) Highest possible MTF using step-and-shoot acquisition mode and filtered backprojection. (Middle) MTF in helical mode and reconstructed with 
Fig. 2 Images of the Teflon rod at different distances from the centre for the UHRCT. The rod imaged by the MDCT at the isocentre of the MDCT system is shown as reference
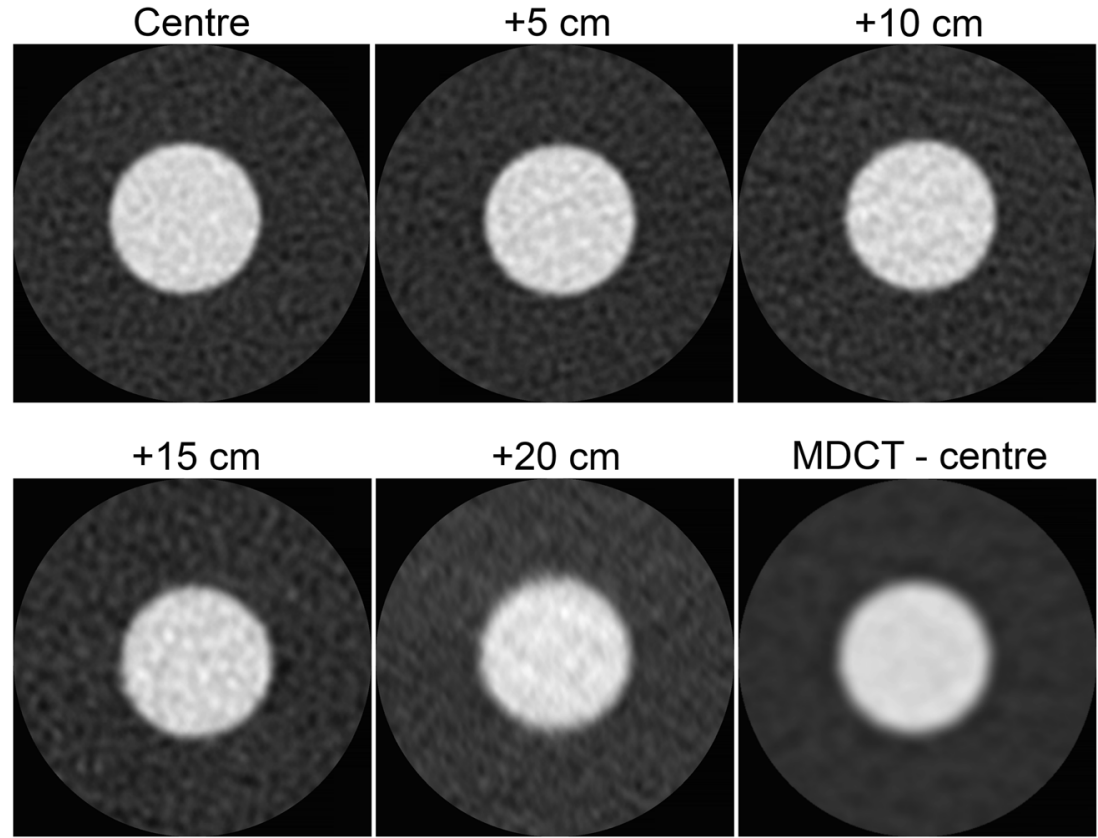

Fig. 3. The peak frequency of the HR and SHR was $0.11 \mathrm{lp} /$ $\mathrm{mm}$. For the NR mode and the MDCT, the peak frequencies were $0.08 \mathrm{lp} / \mathrm{mm}$ and $0.09 \mathrm{lp} / \mathrm{mm}$, respectively.

\section{CT number deviation}

CT numbers were found to be all within the expected range as defined in the ACR protocol [16] except for the LDPE in NR mode (Table 3), which was somewhat lower than expected and lower compared with the SHR and HR modes ( -81 versus - 87)

\section{Low-contrast visibility}

The low-contrast visibility is best for the MDCT. The $0.5 \%$ contrast objects were less visible in the UHRCT NR mode

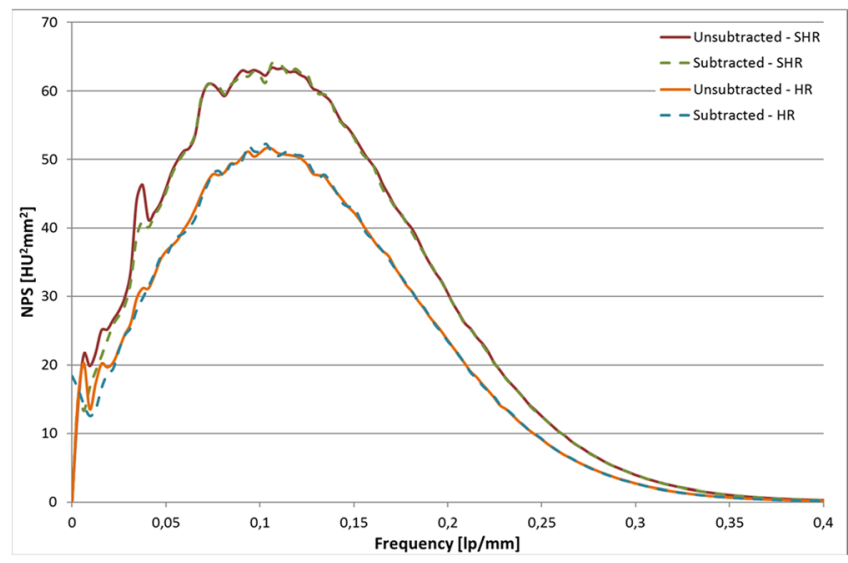

images than in the MDCT ones. For the UHRCT SHR and HR modes compared with MDCT, the edges of the lowcontrast object were more sharply delineated (Fig. 4). In Tables 4 and 5 , the number of visible contrast objects is given. The extra dose necessary for the UHRCT NR mode to have same low-contrast object visibility was $14 \%$. For the HR and SHR modes, this dose increase was approximately $23 \%$.

\section{Discussion}

In this study evaluating the physical properties of UHRCT, we found that spatial resolution in SHR and HR modes is about twice as high as that of the MDCT at the isocentre. Spatial resolution away from the isocentre remains higher than the central resolution in $\mathrm{MDCT}$, except at the outermost

Fig. 3 Subtracted and unsubtracted NPS for the UHRCT in (left) HR and SHR modes and (right) for the NR mode and MDCT. Images are reconstructed using AIDR 3D enhanced reconstruction technique with FC08 filter kernel 
Table 3 The CT numbers resulted from different materials and scan modes. Used phantoms and expected range by the ACR [17] is given in the last column

\begin{tabular}{llcccc}
\hline CT number & & & & \\
\hline Material & Phantom & SHR (HU) & HR (HU) & NR (HU) & Exp. range (HU) \\
\hline Air & Catphan 500 & -981 & -982 & -976 & -1005 to -970 \\
LDPE & Catphan 500 & -87 & -87 & -81 & -107 to -84 \\
Water & 320 mm water phantom & -1.0 & -0.9 & -0.4 & -7 to 7 \\
Acrylic & Catphan 500 & 125 & 124 & 127 & 110 to 135 \\
Teflon & Catphan 500 & 898 & 897 & 920 & 850 to 970 \\
\hline
\end{tabular}
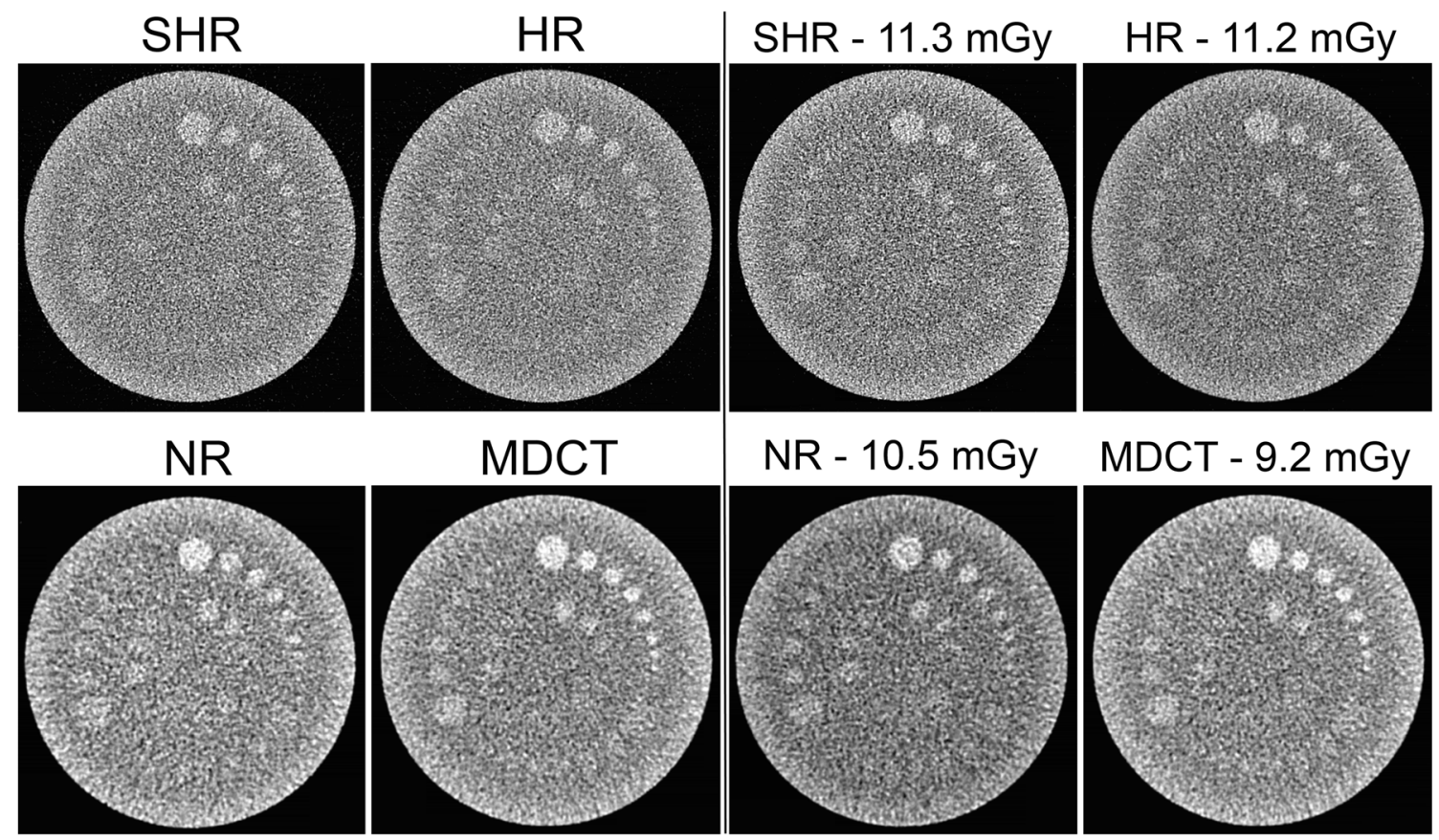

Fig. 4 Images of the Catphan low-contrast objects in the CTP515 module acquired in NR, HR and SHR modes of the UHRCT and on the MDCT (left) at a $\mathrm{CTDI}_{\mathrm{vol}}$ of $9.1 \mathrm{mGy}$ and (right) for the same low-contrast

periphery. In Fig. 5, an example of separate abdominal scans of the same patient on MDCT and on UHRCT, using the HR mode, is shown. In this figure, it can be seen that the UHRCT acquisition results in a better delineation of structures.

The finding that resolution falls off towards the periphery in UHRCT is a known phenomenon and generally observed in $\mathrm{CT}$. This drop in resolution as a function of distance from the centre is likely caused by the finite number of views and focal

Table 4 Number of visible supra slice contrast objects in the Catphan CTP515 module

\begin{tabular}{lllll}
\hline Contrast (\%) & SHR (HU) & HR (HU) & NR (HU) & MDCT (HU) \\
\hline 1 & 7 & 8 & 7 & 8 \\
0.5 & 6 & 5 & 5 & 7 \\
0.3 & 2 & 2 & 3 & 3 \\
\hline
\end{tabular}

detectability, the $\mathrm{CTDI}_{\mathrm{vol}}$ is noted. Note that the window width is not the same for all images; for the SHR and HR modes, it is 60 (left) and 50 (right) and for the NR and MDCT it is 30

spot size. In UHRCT, the relative drop in resolution is comparable to that of other systems [17-19].

As a result of the non-linear behaviour of the hybrid-IR reconstruction technique, the noise magnitudes for the highresolution modes are lower than would be expected given the noise magnitude in NR mode and of the MDCT. Appendix B shows the noise magnitude and texture as a function of dose for hybrid-IR and FBP reconstruction techniques for the

Table 5 Number of visible sub slice contrast objects in the Catphan CTP515 module

\begin{tabular}{lllll}
\hline Z-axis length (mm) & SHR (HU) & HR (HU) & NR (HU) & MDCT (HU) \\
\hline 7 & 2 & 2 & 2 & 3 \\
5 & 1 & 2 & 2 & 2 \\
3 & 0 & 0 & 1 & 1 \\
\hline
\end{tabular}




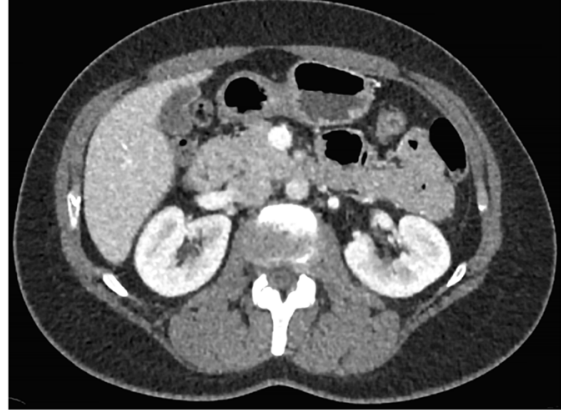

MDCT, AIDR 3D

DLP: 109 mGy.cm

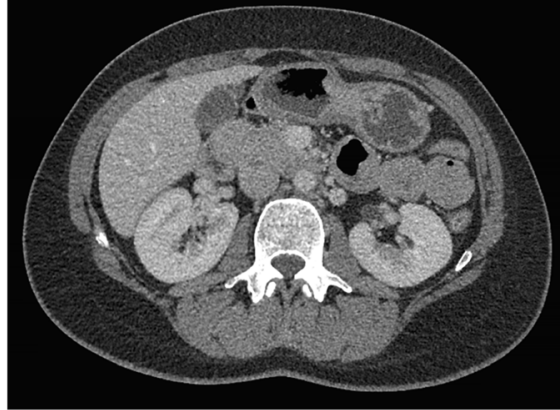

UHRCT, AIDR 3D

DLP: $361 \mathrm{mGy} . \mathrm{cm}$

Fig. 5 Three axial venous phase contrast enhanced abdominal follow-up CT scans of a female patient with a resected stomach tumour at different time-points. (Left) MDCT at $100 \mathrm{kV}$ with hybrid-iterative reconstruction (middle) UHRCT with HR mode at $120 \mathrm{kV}$ with hybrid-iterative reconstruction and (right) UHRCT with HR mode at $120 \mathrm{kV}$ with deep learning image reconstruction. Despite different contrast timing and tube potential

different resolution modes. It can be seen that for the former technique noise magnitude is rather constant over a large range of dose values. However, noise structure becomes grainier with decreasing dose, while with FBP, noise structure remains the same. Using hybrid-IR, resolution is maintained up to a certain point, beyond which resolution is affected too. As expected, this effect is not observed using FBP reconstruction.

The NR mode of UHRCT is comparable with the MDCT with respect to CT number, resolution and uniformity. Using an abdominal soft tissue kernel, the noise in NR mode has a lower magnitude than with the MDCT and lower highfrequency components. This might be caused by the iterative reconstruction algorithm using different internal optimisations and suppressing more high-frequency noise in NR mode on the UHRCT compared with on the MDCT, although the user settings are the same for both reconstructions.

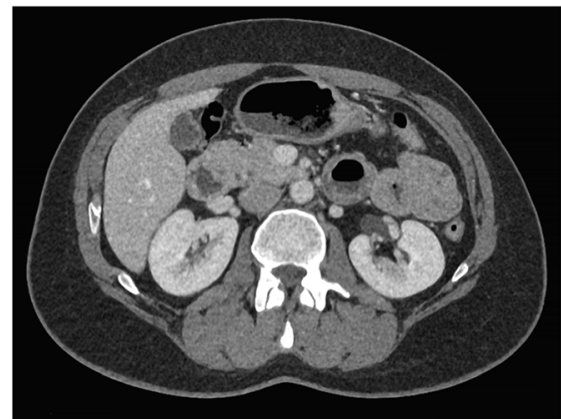

UHRCT, AiCE

DLP: 223 mGy.cm

compared with MDCT, the UHRCT using AIDR has better delineation of (retroperitoneal) structures, but more (fine grained) noise. Using deep learning image reconstruction with UHRCT results in even better delineation of the retroperitoneal structures and mesenteric vessels, with less perceived noise than in the other scans

In SHR and HR modes, the CTDI ${ }_{\mathrm{vol}}$ is higher than that for the NR mode for the same tube settings. This is caused by this UHRCT changing the wedge filter automatically when one of the high-resolution modes is used, resulting in somewhat softer spectra in the high-resolution modes.

Low-contrast detectability is a little lower in the NR mode compared with MDCT, but only a slight dose increase results in the same low-contrast detectability. At our institution, radiation exposure of abdominal MDCT scans is below the achievable levels used in many countries [20,21]; the median (1st and 3rd quartiles) of the $\mathrm{CTDI}_{\mathrm{vol}}$ and DLP used clinically over the last year on our MDCT was $3 \mathrm{mGy}(2.3-4.8 \mathrm{mGy})$ and $155 \mathrm{mGy} \mathrm{cm}$ (116-252 mGy cm), respectively. The slight dose increase necessary to achieve the same low-contrast detectability with the UHRCT in high-resolution modes will not raise

Fig. 6 Two different right ear bone kernel CT scans of fenestral otosclerosis as a subtle, focal area with lucency in front of the stapedial footplate (circle). (Left) MDCT and (right) UHRCT in HR mode, both using hybrid-iterative reconstruction. The UHRCT scan contains less noise and enables better delineation of the stapes (arrow), the trabeculae of the medullary bone of the mastoid, the bony otic capsule and the focal lucency (circle) in this capsule representing the disease
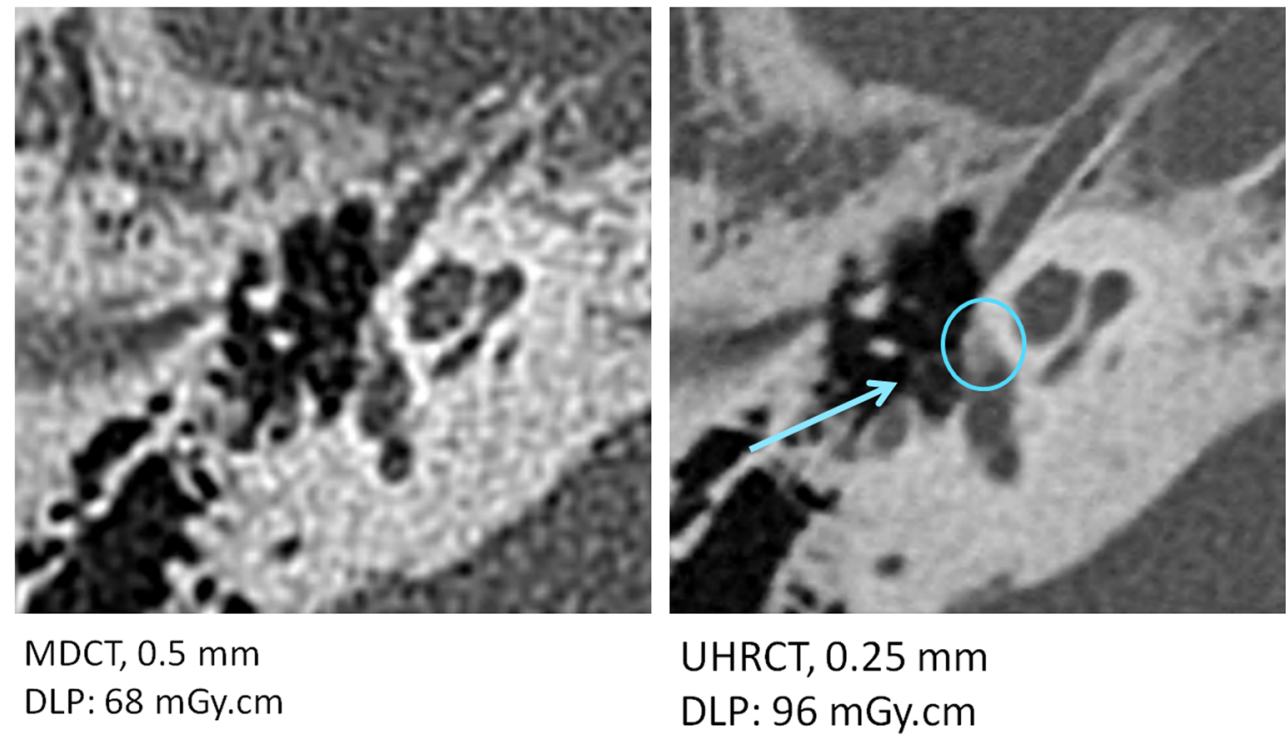

UHRCT, $0.25 \mathrm{~mm}$

DLP: 96 mGy.cm 
the dose above achievable levels of the diagnostic reference levels of $11 \mathrm{mGy}$ and $550 \mathrm{mGy} \mathrm{cm}$.

The low-contrast detectability of the high-resolution modes is lower than that of the MDCT at the same CTDI ${ }_{\mathrm{vol}}$; to make it comparable, an extra dose of about $23 \%$ is needed. However, in clinical practice, the dose used in HR mode is about three times higher than that for NR mode (see also Fig. 5). This is due to the fact that the automatic exposure control aims to keep the noise magnitude constant assuming FBP reconstruction. Since in HR mode the in-plane resolution is twice as high compared with that in the MDCT, it would be expected that a fourfold increase in dose would be needed. The lower observed factor is, in addition to other optimisations, mainly due to a more efficient detector layout, with reduced septa thickness. Lowering the dose to a level that would result in the same low-contrast detectability would lead to a level of high-frequency noise that would probably be unacceptable to radiologists. However, a clinical performance-based assessment of the dose increase required with HR mode has not yet been performed. New deep learning reconstruction techniques (AiCE reconstruction, Canon Medical Systems Corporation) [22] aim to lower the noise while maintaining resolution. In our experience, this technique has a higher impact on higher noise conditions, such as in HR mode at low dose, than in NR mode. Therefore, it allows to achieve scans in HR mode at half the achievable level of the DRL (see Fig. 5).

Our study has several limitations. First, most measurements were performed with an abdominal soft tissue kernel only. Although it is not a high-resolution kernel, it is the most commonly used kernel at our institution. As the system resolution is higher than the resolution at abdominal settings, it is expected that high-resolution kernels could also benefit from the higher resolution capabilities. For example, Fig. 6 shows an inner ear scan reconstructed with a high-resolution kernel. The additional detail in the UHRCT reconstruction, enabling better delineation of the structures, can be appreciated.

Second, the low-contrast measurements were only performed in a qualitative fashion without performing an extensive observer study. Low-contrast details were evaluated while the observer knew where they should be. However, this method is expected to result in a good first-order estimation of the low-contrast capabilities, since any bias will probably affect the results in the same manner for all conditions. More elaborate studies would need to be performed to study lowcontrast visibility in more detail.

Finally, some caution should be used when interpreting linear metrics, such as the MTF and NPS, when evaluating images reconstructed with non-linear algorithms, such as the AIDR3D algorithm used here. The resulting spatial resolution and noise characteristics in different areas containing signals of different characteristics, even across the same image, could vary, and not be well represented by a single MTF or NPS. However, having performed this comparison across acquisition modes and between the two systems using the same testing tools and phantoms does ensure that the relative differences, and similarities, in performance found are reflective of the actual system/algorithm capabilities.

In conclusion, the HR and SHR modes of the UHRCT system result in double the in-plane spatial resolution of the MDCT system, while the NR mode is comparable to that of the MDCT. The trade-off is that about $23 \%$ more dose is needed for the same low-contrast detectability. Upcoming deep learning reconstruction techniques are promising in lowering the current clinical dose penalty while maintaining the spatial resolution.

Funding information This study has received funding by Canon Medical Systems Corporation.

\section{Compliance with ethical standards}

Guarantor The scientific guarantor of this publication is Ioannis Sechopoulos.

Conflict of interest The authors of this manuscript declare relationships with the following companies: Canon Medical Systems Corporation. The study data and results were generated and controlled at all times by the research personnel at Radboud University Medical Center, with no influence from Canon.

Statistics and biometry No complex statistical methods were necessary for this paper.

Informed consent Written informed consent was not required for this study because it was a phantom study.

Ethical approval Institutional Review Board approval was not required because it was a phantom study.

\section{Methodology \\ - Experimental \\ - Performed at one institution}

Open Access This article is licensed under a Creative Commons Attribution 4.0 International License, which permits use, sharing, adaptation, distribution and reproduction in any medium or format, as long as you give appropriate credit to the original author(s) and the source, provide a link to the Creative Commons licence, and indicate if changes were made. The images or other third party material in this article are included in the article's Creative Commons licence, unless indicated otherwise in a credit line to the material. If material is not included in the article's Creative Commons licence and your intended use is not permitted by statutory regulation or exceeds the permitted use, you will need to obtain permission directly from the copyright holder. To view a copy of this licence, visit http://creativecommons.org/licenses/by/4.0/.

\section{References}

1. Rybicki FJ, Otero HJ, Steigner ML et al (2008) Initial evaluation of coronary images from 320-detector row computed tomography. Int J Cardiovasc Imaging 24:535-546. https://doi.org/10.1007/s10554008-9308-2 
2. Duan X, Wang J, Leng S et al (2013) Electronic noise in CT detectors: impact on image noise and artifacts. AJR Am J Roentgenol 201:W626-W632. https://doi.org/10.2214/AJR.12.10234

3. Hata A, Yanagawa M, Honda O et al (2018) Effect of matrix size on the image quality of ultra-high-resolution CT of the lung. Comparison of $512 \times 512,1024 \times 1024$, and $2048 \times 2048$. Acad Radiol 2048:1-8. https://doi.org/10.1016/j.acra.2017.11.017

4. Yoshioka K, Tanaka R, Takagi H et al (2017) Ultra-high-resolution CT angiography of the artery of Adamkiewicz: a feasibility study. Neuroradiology:1-7. https://doi.org/10.1007/s00234-017-1927-7

5. Meijer FJA, Schuijf JD, de Vries J, Boogaarts HD, van der Woude WJ, Prokop M (2019) Ultra-high-resolution subtraction CT angiography in the follow-up of treated intracranial aneurysms. Insights Imaging 10:4-9. https://doi.org/10.1186/s13244-019-0685-y

6. Onishi H, Hori M, Ota T et al (2018) Phantom study of in-stent restenosis at high-spatial-resolution CT. Radiology 289:255-260. https://doi.org/10.1148/radiol.2018180188

7. Gupta R, Grasruck M, Suess C et al (2006) Ultra-high resolution flat-panel volume CT: fundamental principles, design architecture, and system characterization. Eur Radiol 16:1191-1205. https://doi. org/10.1007/s00330-006-0156-y

8. Gupta R, Cheung AC, Bartling SH et al (2008) Flat-panel volume CT: fundamental principles, technology, and applications. Radiographics 28:2009-2022. https://doi.org/10.1148/rg. 287085004

9. Greschus S, Kiessling F, Lichy MP et al (2005) Potential applications of flat-panel volumetric CT in morphologic, functional small animal imaging. Neoplasia 7:730-740. https://doi.org/10.1593/neo. 05160

10. Ross W, Cody DD, Hazle JD (2006) Design and performance characteristics of a digital flat-panel computed tomography system. Med Phys 33:1888-1901. https://doi.org/10.1118/1.2198941

11. Flohr TG, Stierstorfer K, Süss C, Schmidt B, Primak AN, McCollough CH (2007) Novel ultrahigh resolution data acquisition and image reconstruction for multi-detector row CT. Med Phys 34: 1712-1723. https://doi.org/10.1118/1.2722872

12. Imai Y, Nukui M, Ishihara Y et al (2009) Development and performance evaluation of an experimental fine pitch detector multislice CT scanner. Med Phys 36:1120-1127. https://doi.org/10.1118/1. 3086117

13. Leng S, Yu Z, Halaweish A et al (2016) Dose-efficient ultrahighresolution scan mode using a photon counting detector computed tomography system. J Med Imaging (Bellingham) 3:043504. https://doi.org/10.1117/1.JMI.3.4.043504

14. Willemink MJ, Persson M, Pourmorteza A, Pelc NJ, Fleischmann D (2018) Photon-counting CT: technical principles and clinical prospects. Radiology 289:293-312. https://doi.org/10.1148/radiol. 2018172656

15. The Phantom Laboratory (2014) Catphan 500 and 600 Manual. Available via https://www.uio.no/studier/emner/matnat/fys/ nedlagte-emner/FYS4760/h07/Catphan500-600manual.pdf

16. American College of Radiology (ACR) (2017) Computed tomography: quality control. Available via https://www.acr.org/-/media/ ACR/NOINDEX/QCManuals/CT_QCManual.pdf

17. Hara T, Ichikawa K, Sanada S, Ida Y (2010) Image quality dependence on in-plane positions and directions for MDCT images. Eur J Radiol 75:114-121. https://doi.org/10.1016/j.ejrad.2009.03.060

18. Cruz-Bastida JP, Gomez-Cardona D, Li K et al (2016) Hi-res scan mode in clinical MDCT systems: experimental assessment of spatial resolution performance. Med Phys 43:2399-2409. https://doi. org/10.1118/1.4946816

19. Rubert N, Szczykutowicz TP, Ranallo F (2016) Improvement in CT image resolution due to the use of focal spot deflection and increased sampling. J Appl Clin Med Phys 17:6039. https://doi.org/ 10.1120/jacmp.v17i3.6039

20. Kanal KM, Butler PF, Sengupta D, Bhargavan-Chatfield M, Coombs LP, Morin RL (2017) U.S. diagnostic reference levels and achievable doses for 10 adult $\mathrm{CT}$ examinations. Radiology 284:120-133. https://doi.org/10.1148/radiol.2017161911

21. Roch P, Célier D, Dessaud C, Etard C (2018) Using diagnostic reference levels to evaluate the improvement of patient dose optimisation and the influence of recent technologies in radiography and computed tomography. Eur J Radiol 98:68-74. https://doi. org/10.1016/j.ejrad.2017.11.002

22. Akagi M, Nakamura Y, Higaki T et al (2019) Deep learning reconstruction improves image quality of abdominal ultra-highresolution CT. Eur Radiol. https://doi.org/10.1007/s00330-01906170-3

Publisher's note Springer Nature remains neutral with regard to jurisdictional claims in published maps and institutional affiliations. 